\title{
PROTOCOLO DE INVESTIGACION: CONSTRUCCIÓN DE GÉNERO EN ESCOLARES CHILENOS, FUNDAMENTANDO EL CUIDADO
}

\section{RESEARCH PROTOCOL: GENDER BUILDING IN CHILEAN SCHOOLCHILDREN, BASING THE CARE}

\author{
Daniela Montecinos Guiñez ${ }^{1}$ \\ Bárbara Sandoval Erices ${ }^{1}$ \\ Patricia Soto Malabrigo ${ }^{1}$ \\ Flora Andrade Uribe ${ }^{1}$ \\ Amalia Silva Galleguillos ${ }^{1}$ \\ Esmérita Opazo Morales ${ }^{1}$ \\ Mirliana Ramírez Pereira ${ }^{2}$
}

${ }^{1}$ Departamento de Enfermería, Facultad de Medicina, Universidad de Chile.

${ }^{2}$ Autor para correspondencia. mirliana@ uchile.cl. Departamento de Enfermería. Universidad de Chile.

\section{RESUMEN}

Introducción: El proceso de construcción de la identidad de género es un fenómeno socialmente producido y sujeto a influencias que ejercen los distintos espacios de inserción. Es prioritario trabajar esta temática desde la infancia, para establecer relaciones flexibles y equitativas, donde lo femenino y masculino no sea excluyente, ni fuentes de conflicto.

Objetivos: La presente investigación tiene por objetivo comprender las representaciones sociales de estereotipos y roles de género en niñas/os en edad escolar, y proponer estrategias para gestar procesos docentes que incluyan el abordaje de la temática.

Métodos: Mediante la metodología cualitativa, bajo el paradigma post positivista, con enfoque teórico metodológico de etnografía focal. El estudio se realizará por medio del análisis de contenido visual y narrativa. La selección de los participantes será mediante un muestreo por conveniencia y corresponderá a escolares de quinto año de enseñanza básica en 
una escuela municipal de una comuna de Recoleta de la ciudad de Santiago de Chile. Los participantes deberán tener más de diez años, asentir su participación y contar con el consentimiento firmado por sus padres o tutores legales.

Palabras claves: Enfermería, Género, Salud Pública

\section{ABSTRACT}

Introduction: The process of building gender identity is a socially produced phenomenon and subject to influences exerted by the different insertion spaces. It is a priority to work on this issue from childhood, to establish flexible and equitable relationships, where the feminine and masculine are not exclusive, nor sources of conflict.

Objectives: This research aims to understand the social representations of stereotypes and gender roles in school-age girls and boys, and to propose strategies to create teaching processes that include the addressing of the subject.

Methodology: Using qualitative methodology, under the post-positivist paradigm, with a methodological theoretical approach of focal ethnography. The study will be carried out through the analysis of visual and narrative content. The selection of the participants will be by means of a convenience sampling and will correspond to schoolchildren who attend the fifth year of basic education in a municipal school in the district of Recoleta, Santiago city, Chile. The participants must be more than ten years old, agree to their participation and have the consent signed by their parents or legal guardians.

Keywords: Nursing, Gender, Public Health 


\section{INTRODUCCIÓN}

Al hablar de género se considera a las características de mujeres y hombres definidas por la sociedad, como roles o normas y las relaciones existentes entre ellos ${ }^{1}$. El proceso de construcción de la identidad de género es un fenómeno socialmente producido y sujeto a las diversas influencias que ejercen los distintos espacios de inserción, donde las personas interaccionan en su vida cotidiana ${ }^{2,3}$. Las sociedades, históricamente han establecido la existencia de diferencias entre ser hombre y ser mujer, proponiendo la forma en que deben definirse, las acciones que pueden o deben realizar, sus sentimientos o pensamientos, como también que pueden esperar en torno a sus vidas y la forma de relacionarse entre ambos géneros ${ }^{1}$.

Esta diferencia entre los géneros ha sido entendida como una jerarquía moral, donde los hombres suelen representar mayor superioridad ante las mujeres. A su vez, se han destinado ciertos lugares que pertenecen a cada género, donde el espacio doméstico asociado a la crianza y la reproducción se ha atribuido a las mujeres, mientras que áreas públicas donde se obtiene el poder y la producción desde lo laboral, a los hombres ${ }^{3}$.

Las relaciones de género son multidimensionales y dinámicas, se van formando y cambiando según varios escenarios tanto de la vida personal como colectiva, ya sean materiales, psíquicos, institucionales, económicos, políticos y culturales, y todo en su conjunto definirá las condiciones con las cuales las personas forman sus identidades y desarrollan sus relaciones sociales de género ${ }^{4}$.

Investigaciones centradas en las niñas y los niños resaltan que la formación de la identidad de género empieza a desarrollarse a edades muy tempranas ${ }^{2}$. Es por este motivo que se concibe la presente investigación, considerando que la sociedad chilena posee una fuerte presencia de estereotipos y roles socialmente asociados a un determinado género, justificando determinadas conductas que afectan a hombres y mujeres, y se traducen en distintos niveles de violencia que repercuten en las relaciones de pareja, laborales, familiares, entre otras, con una fuerte carga emocional para toda la sociedad, repercutiendo en la salud mental ${ }^{3}$. Según la Encuesta Nacional de Salud del $2009^{5}$, un 17,2\% de la población chilena mayor a 15 años ha presentado síntomas depresivos en el último año y es dos veces más frecuente la depresión en mujeres ${ }^{6}$. Sumado a lo anterior, Chile se encuentra entre los países 
miembros de la OCDE $^{7}$ con mayores tasas de suicidio (13,3 por 100.000 habitantes) y al desagregar por sexo, la tasa de mortalidad es cuatro veces mayor para los hombres que para las mujeres ${ }^{8,9}$.

Por lo tanto, es pertinente trabajar este tema desde la infancia con el propósito de lograr que los géneros tiendan a establecer relaciones flexibles y equitativas respecto al otro, de manera que en el futuro las actuales características de lo femenino y masculino no sean excluyentes, ni fuentes de conflicto entre hombre y mujeres, así como construir desde la infancia normas de género más saludables, que en definitiva constituyen la base del comportamiento humano, incluida la conducta en salud. Es por todo ello, que esta investigación tiene por objetivo:

- Comprender las representaciones sociales de estereotipos de roles de género en niños y niñas de edad escolar

- Proponer estrategias para gestar procesos docentes que incluyan el abordaje de la temática del género con la utilización de metodologías participativas.

Esta investigación forma parte del proyecto "Espejuelos para el género". El cual nace en Cuba el año 2012, como iniciativa de los integrantes de la Red de Enfermería en Salud Infantil (ENSI-Cuba), la organización de Pioneros "José Martí”, la Red de Género y Salud Colectiva y la Facultad de comunicación de la Universidad de la Habana con apoyo de la Organización Panamericana de Salud (OPS), quienes decidieron llevar a cabo un proyecto de investigación acción participación en las escuelas primarias del Municipio Plaza de la Revolución de la Habana, Cuba. El proyecto pertenece a los estudios multicéntricos que desarrolla la Red Internacional de Enfermería en Salud Infantil, donde participan además de Chile, Cuba, Brasil, México, España, Panamá y Uruguay.

El proyecto es justificado por su contribución a distintos compromisos de la OPS. En primer lugar, es un indicador de desarrollo humano. A la vez, contribuye al logro de los Objetivos de Desarrollo del Milenio (ODM) especialmente al número tres, que como meta propone eliminar las desigualdades entre los sexos en la enseñanza primaria y secundaria ${ }^{10}$. Junto con esto, para la OPS/OMS uno de los desafíos para la cooperación técnica en temas de género y salud es el trabajo inter programático, interinstitucional, elevar el conocimiento sobre género, empoderar a los decisores y compartir buenas prácticas en género, oportunidad 
para un mayor intercambio, difusión y aplicación en la cooperación entre los países ${ }^{1}$. A nivel país, contribuye con el objetivo estratégico número cinco de los Objetivos Sanitarios de la Década que busca reducir las inequidades en salud de la población a través de la mitigación de los efectos que producen los determinantes sociales y económicos de la salud ${ }^{11}$.

Además, busca incentivar la formación de niñas y niños como promotores de la equidad de género, desafío que exige trabajo intersectorial y multidisciplinario. Por tanto, implica que la salud de las personas sea vista como una dimensión integral del ser humano y como un objetivo común y compartido.

La igualdad de género en la salud significa que mujeres y hombres se encuentran en igualdad de condiciones para ejercer plenamente sus derechos y su potencial para estar sanos, contribuir al desarrollo sanitario y beneficiarse de los resultados. Además, significa una distribución justa de los beneficios, el poder, los recursos y sus responsabilidades. (12) Este concepto reconoce que entre mujeres y hombres hay diferencias en cuanto a las necesidades en materia de salud, en el acceso y control de los recursos, y que estas diferencias deben abordarse para corregir los desequilibrios existentes ${ }^{13,14}$.

El género es un determinante social de salud (DSS), y en este contexto se alude a las inequidades en función del género que son injusta, inevitable y prevenibles. Según Sen et al ${ }^{15}$, las relaciones de género constituyen la raíz de la causa de las inequidades de género y una de las DSS más influyentes, que incluyen la segregación por género, discriminación por sueldo, expectativas respecto a la conducta sexual y la violencia.

Como se desprende del párrafo anterior, de ahí la importancia del estudio de las representaciones sociales con metodologías cualitativas, construyendo desde las niñas y los niños, y no desde los preconceptos de los investigadores.

\section{METODOLOGÍA}

Esta investigación se conducirá según el paradigma cualitativo, el cual centra su atención en comprender los significados que los sujetos infieren sobre las acciones y conductas sociales, la investigación cualitativa permite comprender los significados, otorgando una importancia contextual, pero sin perder de vista los aspectos particulares de la situación. Esto se relaciona directamente con la idea de que es fundamental la búsqueda de 
sentido, con el fin de lograr la comprensión de los fenómenos, siendo incluso más importante que la explicación de un fenómeno particular. De esto se desprende que los significados son construidos socialmente por individuos que interactúan con su mundo particular, en lugar de la creencia que la verdad es una cualidad singular permanente y no modificable.

El enfoque teórico metodológico que se utilizará será la etnografía focalizada. La etnografía es uno de los métodos de investigación cualitativa más antiguos, surge en la antropología del siglo XIX. La etnografía focalizada se basa en la premisa de que según Mayan en Wall ${ }^{16}$ ya no se necesita viajar a lugares alejados para estudiar la cultura, ya que esta no se define sólo desde la geografía, sino que las culturas y subculturas están en todas partes, siendo especialmente relevante cuando se realizan investigaciones sociales aplicadas en poblaciones fragmentadas y especializadas.

Como referente teórico se trabajará con Janice Morse ${ }^{17}$, quien propone que para realizar etnografía no se requiere de un tiempo prolongado para lograr los objetivos de la investigación, en esta situación se investiga sólo un aspecto de la cultura en un corto periodo de tiempo, como es en este caso, las representaciones sociales de género.

Para el desarrollo de la iniciativa se considerarán tres etapas de trabajo:

I. Iniciación: correspondiente a la conformación del equipo, inducción metodológica, formulación del proyecto y presentación al Comité de Ética.

II. Investigación: etapa de recolección de datos mediante el desarrollo de cuatro talleres.

III. Evaluación: periodo de análisis y discusión de los datos recabados, escritura de informes, artículos científicos y creación de maleta pedagógica.

La presente publicación da cuenta del trabajo desarrollado durante la primera etapa, correspondiente a la formulación del proyecto.

Es importante mencionar que el equipo de investigación dará a conocer los resultados de la investigación a niños (con un lenguaje adecuado para su edad), apoderados y docentes, como una forma de cumplir estándares de calidad en investigación cualitativa, quedando abierta la posibilidad que el Departamento de Enfermería de la Universidad de Chile realice capacitaciones en el tema. 


\section{Participantes:}

Se considerará para la aplicación de la técnica una muestra intencionada no probabilística, es decir un muestreo secuencial teóricamente conducido, donde la selección de los participantes será conducida por la representatividad ${ }^{18}$.

La muestra cualitativa estará compuesta por 45 niños entre 10 y 13 años que cursen quinto básico, pertenecientes a una escuela municipal de una comuna del sector norte de la ciudad de Santiago de Chile, durante el segundo semestre del 2017.

Los participantes deberán cumplir los siguientes criterios de inclusión:

- Estudiantes de quinto año básico, que deseen participar.

- $\quad$ De edad mayor o igual de diez años.

- Que cuenten con el consentimiento informado firmado por sus padres o tutores legales.

- Que cuenten con asentimiento informado firmado por escolares.

\section{Técnicas de recolección de datos:}

La recolección de los datos se realizará mediante el desarrollo de cuatro talleres en fechas acordadas con la escuela.

Cada taller se realizará dentro del horario de clases, durante el bloque de orientación y con una duración de una hora, a fin de no interrumpir la planificación académica. Serán guiados por los investigadores en compañía del profesor jefe u orientador.

Como técnica de recolección de datos se utilizarán dibujos realizados por los estudiantes, escrituras de cartas breves y listados de preferencias musicales. 
A continuación, se presentan los talleres que formarán parte de la etapa de investigación del proyecto.

- Taller 1: Animación e integración

- Taller 2: Artes Visuales. Considera la realización de tres dibujos que respondan las preguntas:

- ¿Cómo soy?

- ¿Cómo debo ser como niño o niña?

- ¿Cómo no debo ser?

- Taller 3: Narrativa: Considera la escritura de una carta, donde relaten:

- ¿Qué hago después de clases?

- Taller 4: Música: Considera la escritura de un listado de preferencias musicales, donde relaten:

- ¿Qué canciones escucho? y ¿Por qué?

\section{Metodología de análisis de datos:}

Se realizará análisis de contenido, el cual se entiende como una técnica de interpretación de textos, ya sean escritos, grabados, pintados o filmados. El denominador común de todos estos materiales es su capacidad para albergar un contenido que leído e interpretado adecuadamente abre las puertas al conocimiento de diversos aspectos y fenómenos de la vida social ${ }^{19}$.

El análisis de contenido se basa en la lectura (textual o visual) como instrumento de recogida de información, lectura que a diferencia de la lectura común debe realizarse siguiendo el método científico, es decir, debe ser sistemática, objetiva, replicable, y válida.

Es una técnica que combina intrínsecamente la observación, producción de información y la interpretación de los mismos ${ }^{18}$. El análisis de los elementos visuales se realizará desde la perspectiva de las representaciones sociales de género, considerando elementos como el tipo de líneas, sombras, color, espacio y forma; y los principios de balance, énfasis, contraste, repetición y patrones ${ }^{19}$. 


\section{CRITERIOS ÉTICOS}

En este proyecto se asumen los principios de Exequiel Emanuel, modificados por González Ávila, quien agrega que, para considerarse como ética una investigación cualitativa debe incluir las condiciones de diálogos auténticos.

En este estudio se desarrollarán los requerimientos de valor social o científico, la validez científica, selección equitativa de las personas, proporción favorable del riesgobeneficio, evaluación independiente, consentimiento y asentimiento informado y respecto a las personas inscritas.

El consentimiento informado será dirigido a los padres o tutores y el asentimiento informado a niñas y niños. En ellos se darán a conocer el objetivo principal de la investigación, los beneficios para los estudiantes y la metodología con que se trabajará. También se destacará que la participación del estudio es totalmente voluntaria, siendo un proceso confidencial y que guardará el anonimato, explicitando que, si se desea, se pueden retirar en cualquier momento de la investigación.

La investigación cuenta con la aprobación del comité de Ética de la Facultad de Medicina de la Universidad de Chile, con fecha 11 de Julio del 2017, correspondiente al número de proyecto 087-2017.

Para efectos de esta investigación se utilizarán los criterios de rigor desarrollados por Guba y Lincoln. Los autores mencionan que se debe evaluar la confiabilidad de los procesos y procedimientos en investigación adoptando un marco referencial. Se consideran paralelos a las nociones positivistas de rigor e incluyen la credibilidad, transferibilidad, dependencia y confirmabilidad.

\section{FINANCIAMIENTO:}

El proyecto cuenta con el financiamiento interno del Departamento de Enfermería de la Universidad de Chile.

\section{CONFLICTO DE INTERÉS:}

Las investigadoras declaran no tener conflictos de interés. 


\section{RESULTADOS ESPERADOS}

Se espera poder comprender las representaciones sociales de género en niñas y niños en edad escolar, con el fin de diseñar en conjunto con ellos, estrategias que permitan un cambio en la forma de vincularse con sus pares.

Además, la elaboración de un Informe general y resumen ejecutivo para difusión específica a centros especializados con información de la investigación, y la construcción de una maleta pedagógica digital de buenas prácticas para los niños y niñas en edad escolar, con respecto a temática de género.

\section{REFERENCIAS}

1. Organización Mundial de la Salud. [Internet]. n.d. Género. [Citado el 1 de noviembre de 2016]. Disponible en: http://www.who.int/mediacentre/factsheets/fs403/es/

2. Rivera Y. Socialización, género y medios de comunicación. La Habana: Editorial de la Mujer; 2011.

3. Programa de las Naciones Unidas para el desarrollo. Desarrollo Humano en Chile: Género, los desafíos de la igualdad. [Internet]. Santiago de Chile. 2010. [Citado Abril, 2015]. Disponible en: http://desarrollohumano.cl/idh/download/PNUD_LIBRO.pdf

4. Ministerio de Educación, Gobierno de Chile. Formación en Sexualidad, Afectividad y Género. [Internet]. Santiago de Chile. [citado 1 de nov 2016]. Disponible en: http://portales.mineduc.cl/usuarios/convivencia_escolar/doc/201307221719000.PD

F_formacion_sexualidad.pdf

5. Valdez C, Errázuriz P. Salud mental en Chile: El pariente pobre del sistema de salud. Instituto de Políticas Públicas: Claves de políticas públicas. [Internet]. 2012. [Citado mayo, 2016]. Número $11 . \quad$ Disponible en: http://www.politicaspublicas.udp.cl/media/publicaciones/archivos/350/Salud_Menta 1_en_Chile_El_Pariente_Pobre_del_Sistema_de_Salud.pdf

6. Ministerio de Salud, Gobierno de Chile. Programa de Salud Mental y Psiquiatría: Personas afectadas por Depresión. [Internet]. Chile. [citado 1 de abril 2017]. 
Disponible

en: http://web.minsal.cl/portal/url/item/71e6341aec4aaae2e04001011f017bae.pdf

7. Organización Cooperación Desarrollo Económico (OCDE). Health at a Glance 2013: OECD Indicators. [Internet]. OECD Publishing. 2013. [citado 1 de nov 2017]. Disponible en:

http://www.oecdilibrary.org/docserver/download/8113161e.pdf?expires=1509626285\&id=id\&accna $\underline{\text { me}=\text { guest } \& \text { checksum }=C 8 B 96 C 81905 E 6 F 337 A 9 E 8898 B 5 B 5 A 8 B A}$

8. Pontificia Universidad Católica de Chile, Centro de políticas públicas. Aumento sostenido del suicidio en Chile: un tema pendiente. [Internet]. Santiago de Chile. 2015. [citado 1 de abril 2017]. Disponible en: http://politicaspublicas.uc.cl/wpcontent/uploads/2015/07/N\%C2\%B0-79-Aumento-sostenido-del-suicidio-en-

Chile.pdf

9. Ministerio de Salud, Gobierno de Chile. Programa Nacional de Prevención del Suicidio: Orientaciones para su implementación. Norma administrativa $\mathrm{N}^{\circ} 027$. [Internet]. Chile. 2013. [citado 1 de abril 2017]. Disponible en: http://web.minsal.cl/sites/default/files/Programa_Nacional_Prevencion.pdf

10. Naciones Unidas. Objetivo de desarrollo del Milenio. Informe 2015. [Internet]. Nueva York. 2015. [citado 1 de abril 2017]. Disponible en: http://www.un.org/es/millenniumgoals/pdf/2015/mdg-report-2015_spanish.pdf

11. Ministerio de Salud, Gobierno de Chile. ESTRATEGIA NACIONAL DE SALUD: Para el cumplimiento de los Objetivos Sanitarios de la Década 2011-2020. [Internet]. Chile. 2010. [citado 1 de abril 2017]. Disponible en: http://www.minsal.cl/portal/url/item/c4034eddbc96ca6de0400101640159b8.pdf

12. La Colla, L. Representaciones sociales: una manera de entender las ideas de nuestros alumnos. Revista Electrónica de la Red de Investigación Educativa [Internet]. 2005. [Citado mayo, 2015]. Volumen (1), No.3. Disponible en: http://revista.iered.org/v1n3/pdf/llacolla.pdf

13. Organización Panamericana de la Salud. Hoja Informativa para Programa Mujer, Salud y Desarrollo: Equidad de género en salud. [Internet]. Washington DC, Estados 
Unidos. [Citado mayo, 2015]. Disponible en internet: http://www1.paho.org/Spanish/AD/GE/GEHFactSheetSpanish.pdf?ua=1

14. Organización Panamericana de la Salud. Política de igualdad de género. [Internet]. Washington DC, Estados Unidos. [Citado mayo, 2016]. Disponible en internet: http://www.paho.org/hq/dmdocuments/2009/OPS-politica-de-igualdad-degenero.pdf?ua $=1$

15. Ramos-Pibernus A, Rodríguez-Madera S, Rivera-Segarra E. El género como determinante social de la salud. [Internet]. Boletín Diversidad. Puerto Rico. 2014. [Citado Abril, 2016]. Disponible en: https://www.researchgate.net/profile/Eliut_Rivera-

Segarra/publication/259998157_El_genero_como_determinante_social_de_la_salud $\underline{\text { /links/0a85e52ef9e980dcec000000/El-genero-como-determinante-social-de-la- }}$ salud.pdf

16. Stahlke Wall, S. Focused Ethnography: A Methodological Adaptation for Social Research in Emerging Contexts. Forum Qualitative Sozialforschung / Forum: [Internet]. Qualitative Social Research, 16(1). 2014. [Citado Abril, 2016]. Disponible en: http://www.qualitative-research.net/index.php/fqs/article/view/2182

17. Morse, J. Estilo de etnografía. En: Asuntos críticos en los métodos de investigación cualitativa. Universidad de Antioquia. Medellín: Colombia; 2003. p 185- 211.

18. Miles MB, Huberman AM. Qualitative data analysis. A sourcebook of new methods. Bervely Hills: Sage Publications; [Internet]. 1994. [Citado: 2014 Ene]. Disponible en:

https://books.google.es/books?id=46jfwR6y5joC\&pg=PR12\&lpg=PR12\&dq=Miles \pm MB,+ Huberman+AM.+Qualitative+data + analysis. + A + sourcebook + of + new + meth ods.+Bervely+Hills;+1994\&source=bl\&ots=snCUGHuuKW\&sig=DOheLvidvxmdJ $\underline{\text { iELSmxnSmLAicU\&hl=es\&sa=X\&ved=0CCgQ6AEwAGoVChMIuKOkovTsxgIV }}$ QTI-Ch3-

$\underline{5 \mathrm{AUL} \# \mathrm{v}=\text { onepage \&q=Miles\%20MB\%2C\%20Huberman\%20AM.\%20Qualitative\% }}$ 20data\%20analysis. $\% 2$ 
19. Andréu Abela, J. Las técnicas de análisis de contenido: una revisión actualizada. [Internet]. Fundación Centro Estudios Andaluces, Universidad de Granada, v.10, n. 2, p. 1-34, 2000. [citado 1 de Abril 2017]. Disponible en: $\langle$ http://public.centrodeestudiosandaluces.es/pdfs/S200103.pdf $>$. 